\title{
Microfilaria in Liver Aspirate: A Rare Finding
}

\author{
SR Rana Safia ${ }^{1}$, ZSJ Jairajpuri S Zeeba ${ }^{2}$, Jetley Sujata MD ${ }^{3 *}$, SK Khetrapal Shaan MD ${ }^{4}$
}

${ }^{1}$ Assistant Professor, ${ }^{2}$ Associate Professor, ${ }^{3}$ Professor, ${ }^{4}$ Assistant Professor, Department of Pathology Hamdard Institute of Medical Sciences and Research Jamia Hamdard, New Delhi 110062 India

DOI: $\underline{10.36347 / \text { sjmcr.2020.v08i01.002 }}$

| Received: 10.12.2019 | Accepted: 17.12.2019 | Published: 10.01.2020

*Corresponding author: Sujata Jetley

\section{Abstract}

Filariasis caused by Wuchereria bancrofti is a disease of tropical country like India. The detection is conventionally by demonstration of microfilariae in the peripheral blood smear. However, microfilariae can be incidentally detected in fine needle aspirates of various lesions. Although microfilariae have been reported in cytological specimens of many organs, but it is extremely rare to find microfilaria in cytological smears of liver aspirates [1]. We hereby report a rare case of microfilaria in liver aspirates of a 37 year old male who presented with yellowish discoloration of eyes, urine and malena. Radiological investigations were suggestive of nodular regeneration and hyperplasia in liver. Ultrasound (USG) guided aspirates yielded microfilariae in liver aspirate cytology smears. A final impression of filariasis was made.

Keywords: Wuchereria bancrofti, microfilariae, cytological specimens.

Copyright @ 2020: This is an open-access article distributed under the terms of the Creative Commons Attribution license which permits unrestricted use, distribution, and reproduction in any medium for non-commercial use (NonCommercial, or CC-BY-NC) provided the original author and source are credited.

\section{INTRODUCTION}

Filariasis is a parasitic disease, prevalent worldwide. Wuchereria bancrofti is responsible for $90 \%$ cases of filariasis and is found throughout the tropics especially China, India, Indonesia and some parts of Africa [2, 3]. The methods used for diagnosis of filariasis are demonstration of microfilaria in peripheral blood films, microfilarial antigen detection and demonstration of adult forms on histopathology. In cytological aspirates, microfilariae are usually incidental findings. However, such findings signify the importance of fine needle aspiration cytology (FNAC) in the detection of microfilaria, in cases where they are least suspected clinically $[4,5]$.

\section{Case Report}

A 37 year old male presented in the medicine OPD with pain in back and legs with generalised weakness for the past 2 months. He had yellowish discoloration of eyes and urine and two episodes of passing black, tarry stool in the past 20 days. Patient was apparently alright two months back, when he started complaining of backache, severe in nature radiating to back of thigh. Pain aggravated on walking and changing posture. There was no past history of diabetes mellitus/hypertension, tuberculosis or thyroid disorders. He had a history of chronic intake of alcohol and tobacco chewing with intravenous drug abuse. On general examination, patient was conscious and oriented. Marked palor and icterus were seen, however, there was no sign of edema or cyanosis or portal hypertension. Pulse rate was $80 / \mathrm{min}$, blood pressure114/76mmHg.

Laboratory investigations were done. Hemoglobin was $8.6 \mathrm{~g} / \mathrm{dl}$, total leucocyte count $6700 / \mathrm{mm} 3$, polymorphs $53 \%$, lymphocytes $35 \%$, eosinophils $10 \%$, platelet count was $1.08 \mathrm{lacs} / \mathrm{mm} 3$. Biochemical work up revealed Serum creatinine $1.3 \mathrm{mg} / \mathrm{dl}$, Serum calcium $10.6 \mathrm{mg} / \mathrm{dl}$, Serum Phosphorus 2.9meq/L. HBsAg, HCV, HIV I and II were seronegative. Serum bilirubin was elevated markedly, $9.1 \mathrm{mg} / \mathrm{dl}$ with direct fraction $7.3 \mathrm{mg} / \mathrm{dl}$, SGOT 131 IU/L, SGPT 163IU/L, Serum Alkaline phosphatase 723 IU/L. Stool for occult blood was negative Computerised tomographic scan of abdomen was done, multiple variable sized focal lesions of fatty infiltration were seen in right lobe of liver (largest 55 x $58 \mathrm{~mm}$ ) suggestive of nodular regeneration and hyperplasia in liver. Multiple lytic lesions were seen in the vertebrae. A provisional diagnosis of alcoholic liver disease with collapse of multiple vertebrae was made. An ultrasound guided FNAC was done revealing moderately cellular smears with dispersed population of hepatocytes with few small sized clusters showing moderately abundant cytoplasm and round nuclei with bland nuclear chromatin. Many hepatocytes showed bile pigment in cytoplasm suggestive of bile cholestasis. Background 
showed multiple coiled, sheathed microfilarial forms of Wuchereria bancrofti with free tail ends. (Figure1) Based on the above findings, a diagnosis of microfilariae of $\mathrm{W}$. bancrofti was made on cytological smears. A peripheral blood film was examined and screened thereafter, but was found to be negative for microfilaria. However, before the patient could be followed up, he succumbed to cardiac arrest soon during the course of treatment.

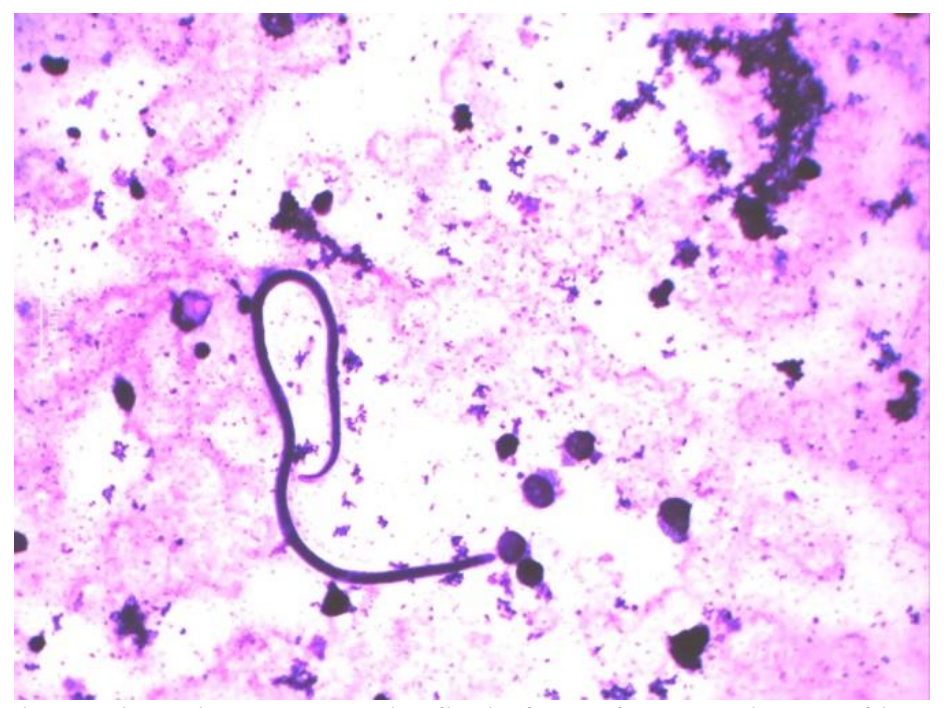

Fig-1: FNAC smear showing multiple coiled, sheathed microfilarial forms of Wuchereria bancrofti alongwith many hepatocytes

\section{DISCUSSION}

Filariasis is a major public health problem in India. The most common species found in India is Wucheria bancrofti. Filariasis is diagnosed either by direct evidence or by indirect immunoallergic tests. FNAC is rarely used in the routine diagnosis of filariasis. But microfilaria has been reported in cytological smears in clinically unsuspected cases. Limited reports are available in the literature, highlighting the importance of FNAC as a tool in the diagnosis of filariasis in the early stages [6]. Microfilariae may not be seen in peripheral blood due to elephantiasis, lymphangitis, and early stages of allergic manifestations and in occult filariasis. In classical presentation of filariasis, lymphangitis is the basic lesion. Apart from lymph nodes, filariasis is seen in lungs, liver and spleen. They have been reported at unusual sites like thyroid, soft tissue, bone marrow, epididymis, lung, breast on FNAC as well as in gastric brushings, cervico-vaginal smears and hydrocele fluid [7].

Microfilariae have been reported in many sites but rarely in liver as reported by Arakeri et al.[8]. A literature review has reported only four cases of microfilariae. Coexistence of microfilaria has also been found with various benign and malignant tumors. Interstingly, they were mostly reported in association with benign or malignant tumors such as cavernous hemangioma of the liver, primary hepatocellular carcinoma and metastatic pancreatic adenocarcinoma [9-11]. It is thought that tumours with rich vascular supply enhance the parasite concentration at the tumour area. Eventually, these blood vessels may rupture leading to the release of parasites into the tumour tissue.
Hence presence of microfilariae in neoplasm is an incidental finding [12]. In the present case, filariasis in liver was diagnosed without coexistent tumors; benign or malignant. The patient was immunocompromised and was a native from Bihar which is an endemic zone for this organism.

Microfilaria circulate in the vascular and lymphatic systems, appear in tissue fluids and exfoliated surface material in case there is lymphatic or vascular obstruction, causing extravasation of blood and release of microfilariae into the blood circulation. Hence lymphatic blockage by scars or tumors and damage to the vessel wall by inflammation, trauma, or stasis can often be a cause of their appearance of these parasites at such unususal sites.

Though FNAC is not used routinely in the diagnosis of filariasis, it can serve as an invaluable tool in the diagnosis of filariasis, especially when there is no clinical suspicion. A cost effective, outpatient procedure like FNAC can help in diagnosing microfilaria and institution of specific treatment.

\section{REFERENCES}

1. Vij Mukul, · Kumari Niraj, Krishnani Narendra. Microfilaria in liver aspiration cytology: an extremely rare finding. Diagn Cytopathol. 2011;39:521-2.

2. Melrose WD. Lymphatic filariasis: New insights into an old disease. Int'l J Parasitol. 2002; 32: $947-$ 60.

3. Kanade US, Dantkale SS, Chaware SA, Narkhede RR, Gadhekar MS. Microfilaria in preauricular 
subcutaneous nodule on cytology: An unusual presentation. JEMDS. 2012 Oct;1:382-6.

4. Jha A, Shrestha R, Aryal G, Pant AD, Adhikari RC, Sayami G. Cytological diagnosis of bancroftian filariasis in lesions clinically anticipated as neoplastic. Nepal Med Coll J. 2008 Jun;10(2):108-14.

5. Sivakumar S. Role of fine needle aspiration cytology in detection of microfilariae: Report of 2 cases. Acta Cytol. 2007; 51:803-6.

6. Dey PR, Radhika SR, Jain A. Microfilariae of Wuchereria bancrofti in a lymph node aspirate. A case report. Acta cytologica. 1993;37(5):745-6.

7. Ahmad SS, Hassan MJ, Akhtar K, Arif SH, Naim M, Rahman K. Microfilariae in testicular fine needle aspiration biopsy. JK Sci. 2008;10(4):199200.
8. Arakeri SU, Yelikar BR. Microfilariae in cytological smears of hepatocellular carcinoma. Journal of Cytology. 2007 Jul 1;24(3):158.

9. Agarwal R, Khanna D, Barthwal SP. Microfilariae in a cytologic smear from cavernous hemangioma of the liver. A case report. Acta cytologica. 1998;42(3):781-2.

10. Arakeri SU, Yelikar BR. Microfilariae in cytological smears of hepatocellular carcinoma. Journal of Cytology. 2007 Jul 1;24(3):158.

11. Mohan S, Andley M, Talwar N, Ravi B, Kumar A. An unusual association with carcinoma pancreas: a case report. Cytopathology. 2005 Aug;16(4):215-6.

12. Gupta S, Sodhani P, Jain S, Kumar N. Microfilariae in association with neoplastic lesion: report of five cases. Cytopathology. 2001; 12:1206. 